\title{
The association between an endothelial nitric oxide synthase gene polymorphism and coronary heart disease in young people and the underlying mechanism
}

\author{
XIAO-JIE CHEN ${ }^{1}$, CHUN-GUANG QIU ${ }^{1}$, XIANG-DONG KONG ${ }^{2}$, SHU-MIN REN ${ }^{2}$, JIAN-ZENG DONG ${ }^{1}$, \\ HE-PING GU $^{1}$, YING-WEI CHEN ${ }^{1}$, HAI-LONG TAO ${ }^{1}$ and JHA SARBESH ${ }^{1}$ \\ Departments of ${ }^{1}$ Cardiology and ${ }^{2}$ Genetics and Prenatal Diagnosis, \\ The First Affiliated Hospital of Zhengzhou University, Zhengzhou, Henan 450000, P.R. China
}

Received May 12, 2017; Accepted November 2, 2017

DOI: $10.3892 / \mathrm{mmr} .2017 .8314$

\begin{abstract}
With the development of molecular biological technology, the association between genes and diseases has drawn increasing attention of researchers; the endothelial nitric oxide synthase (eNOS) gene has been reported to be a candidate gene for cardiovascular disease (CHD). The present study aimed to investigate the association between a polymorphism of eNOS and the risk of CHD in young people ( $\leq 40$ years old), in addition to the underlying mechanism. A total of 234 cases of CHD in young individuals were collected as the CHD group and 228 cases of healthy individuals as the control group. Peripheral blood was collected and the genotype of the eNOS G894T polymorphism was identified by polymerase chain reaction-restriction fragment length polymorphism, the gene frequency was calculated and the distributions of genotype and allele frequency between the two groups were compared. Bioinformatics tools were employed to analyze the differences in the local protein structures of the eNOS G894T polymorphism and the biological mechanism was preliminary discussed. The results demonstrated that there were significant differences in the distribution of genotype frequency and allele frequency of the eNOS G894T gene polymorphism between the CHD group and control group $(\mathrm{P}<0.05)$. The risk of CHD in GT and TT genotypes were higher compared with the GG genotype $(\mathrm{P}<0.05)$. The G894T polymorphism led to Glu298Asp mutation of encoded protein, which is within
\end{abstract}

Correspondence to: Professor Chun-Guang Qiu, Department of Cardiology, The First Affiliated Hospital of Zhengzhou University, 1 East Jianshe Road, Zhengzhou, Henan 450000, P.R. China E-mail: qiuchunguang_xnk@163.com

Abbreviations: CHD, coronary heart disease; eNOS, endothelial nitric oxide synthase; $\mathrm{NO}$, nitric oxide

Key words: endothelial nitric oxide synthase, gene polymorphism, coronary heart disease of young people, G894T, bioinformatics analysis the active site of eNOS, and partial structures of the protein were converted from random coil to $\alpha$-helix. In conclusion, the eNOS G894T gene polymorphism was associated with the occurrence and development of CHD in young people. The potential mechanism is that the G894T polymorphism leads to altered protein structure, which affects the function of eNOS in generating nitric oxide and cardiovascular diastole. The results of the present study suggested a potential target gene for the prevention and treatment of CHD in young people ( $\leq 40$ years old).

\section{Introduction}

Coronary heart disease (CHD) is a type of cardiovascular disease causing by coronary artery atherosclerosis and plaque rupture, leading to the arterial stenosis or occlusion, then resulting in myocardial ischemia or necrosis, which is among the top ten causes of human mortality. At present, the treatment methods of CHD include: Surgical treatment, interventional therapy (stent implantation) and medication (nitric acid ester, $\beta$-blocker, antithrombotic and statins); however, medication is the basis of all treatment (1). With the improvement of the living standards, the incidence and mortality rates of CHD has increased rapidly, particularly within young people ( $\leq 40$ years old) in China $(2,3)$. The earlier incidence of CHD is not only threat to human health, but may burden a patient's families and society; CHD has gained increasing attention of researchers (4). It is well-known that smoking, drinking, obesity, hypertension and metabolic syndrome are risk factors for CHD. For smokers, nicotine in tobacco may damage the vascular endothelium, resulting in the vasospasm and plaques rupture, accelerating the onset of coronary atherosclerosis (5). In addition, previous studies have demonstrated that the functional disturbance of endothelial cells and early-stage atherosclerosis may be caused by reduced levels of nitric oxide (NO), nitric oxide has an important role in the pathogenesis of CHD (6-8). Endothelial NO synthase (eNOS) is the enzyme responsible for the generation of NO in endothelial cell (9-11). Various studies have investigated the association between the eNOS G894T polymorphism and the risks of CHD (12-15). However, to the best of our knowledge, the association 
between eNOS and the risk of CHD in young people is yet to be established. Therefore, in the present study, the association between the eNOS G894T polymorphism and CHD in young individuals, and the influence of the polymorphism on the protein structure and function, were analyzed and the potential biological mechanisms were preliminarily investigated by a series of bioinformatics analyses.

\section{Materials and methods}

Study subjects. A total of 234 cases of young patients with CHD, who received treatment between August 2013 and May 2016 at the Department of Cardiology, The First Affiliated Hospital of Zhengzhou University (Zhengzhou, China) were included in the CHD group, which comprised 198 males and 36 females between the ages of 18 and 40 years old with a mean age of $34.2 \pm 3.7$ years old. All cases were diagnosed with CHD through a coronary angiogram and complied with the following CHD diagnostic criteria specified by the World Health Organization (16): Exhibited symptoms typical of angina; an electrocardiogram identified the previous occurrence of a myocardial infarction; and confirmation by angiography, which is considered the 'gold standard' for the assessment of coronary artery disease. A comprehensive individualized treatment program should be applied to suit the circumstances of each patient. A total of 228 healthy individuals between the ages of 18 and 40 years old who visited the hospital for physical examination and voluntarily agreed to participate in the study during the same period were randomly collected as the control group, which comprised 195 males and 33 females with a mean age of $35.7 \pm 2.6$ years old. All of the participants lived in the Henan province of China and had no blood relationship with each other. The present study was reviewed and approved by the Ethical Inspection Committee of Zhengzhou University and participants in the CHD and control groups volunteered and signed the informed content.

Identifying genotypes bypolymerase chain reaction-restriction fragment length polymorphism (PCR-RFLP). Peripheral blood (4 ml) was collected from each participant, $0.6 \mathrm{mg}$ EDTA-Na was used as an anticoagulant (final concentration was $1.5 \mathrm{~g} / \mathrm{l}$ ) and the genomic DNA was extracted using a Genelute ${ }^{\mathrm{TM}}$ blood genomic DNA kit (Sigma-Aldrich; Merck KGaA, Darmstadt, Germany) according to the manufacturer's protocols. The diagnostic primer for the genotype of the G894T polymorphism was synthesized by Sangon Biotech Company (Shanghai, China): Sense, 5'-GAGATGAAGGCAGGAGACAGT-3'; and anti-sense, 5'-TCCATCCCACCCAGTCAAT-3'. The PCR reaction system $(25 \mu \mathrm{l})$ constituted: Genomic DNA $1.0 \mu \mathrm{l}, 10 \mathrm{x}$ buffer $2.5 \mu \mathrm{l}$, dNTP Mix $2.0 \mu \mathrm{l}$, sense primer $0.2 \mu \mathrm{l}$, anti-sense primer $0.2 \mu \mathrm{l}$, Taq DNA polymerase $0.1 \mu \mathrm{l}$ and deionized water $19 \mu \mathrm{l}$. The amplification procedure: pre-denaturation at $95^{\circ} \mathrm{C}$ or $2 \mathrm{~min} ; 35$ cycles of denaturation at $95^{\circ} \mathrm{C}$ for $30 \mathrm{sec}$, annealing at $58^{\circ} \mathrm{C}$ for $30 \mathrm{sec}$ and extension for $30 \mathrm{sec}$ at $72^{\circ} \mathrm{C}$ and final extension at $72^{\circ} \mathrm{C}$ for $5 \mathrm{~min}$. The PCR products were purified with Gel PCR purification kit (Thermo Fisher Scientific, Inc., Waltham, MA, USA) according to the manufacturer's protocols. PCR-amplified specific fragments of eNOS were obtained. Amplified products $(10 \mu \mathrm{l})$ were digested with $0.5 \mu \mathrm{l}$

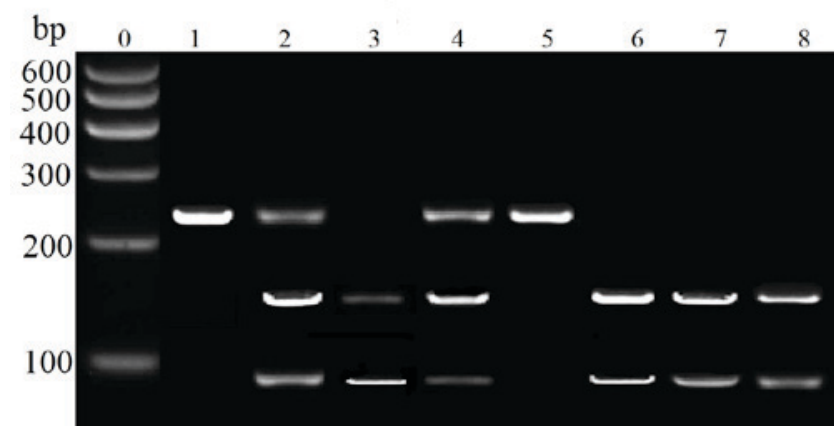

Figure 1. The image of agarose gel electrophoresis for enzyme digestion products. 0: DNA marker, 1-8: the enzyme digestion products in different individuals.

restriction endonuclease Eco24I (Ban II) (Takara, Japan) at $37^{\circ} \mathrm{C}$ for $10 \mathrm{~h}$. The results of enzyme digestion was performed by agarose gel electrophoresis (Fig. 1) and the results demonstrated that wild-type homozygote GG contained 169 and 94 bp fragments, variant homozygote TT contained a single 263 bp fragment and heterozygote GT contained 263 and 169 and 94 bp fragments. The G8494T polymorphism from individuals of the present study had been sequence by PCR-RFLP; in the GG genotype, the G849T locus was at a guanine nucleotide, which could be digested by Eco24I. Within the TT genotype, the G849T locus was at a thymine nucleotide; however, within the GT type, the G849T locus was a guanine or thymine nucleotide.

Bioinformatics analysis on the polymorphism site. Alterations in the amino acid sequences of the eNOS G894T gene polymorphism were analyzed by the Single Nucleotide Polymorphisms and open reading frame databases (National Center for Biotechnology Information (NCBI); https://www.ncbi. nlm.nih.gov/snp/; https://www.ncbi.nlm.nih.gov/orffinder/, respectively), and alterations in the protein structure prior to and following the amino acid sequence mutation were analyzed using the protein modeling tool, QUARK (17). Amino acid sequences consisting of 20 amino acids around the G894T polymorphism site were entered into the QUARK tool to perform local structural modeling of the protein, and structural characteristics prior to and following the mutation were analyzed and compared by $\mathrm{Cn3D}$ 4.3.1 viewer software (NCBI; http://www.ncbi.nlm.nih.gov). Characteristics of the eNOS structural domain were analyzed by SMART v7 (18) and the NCBI Conserved Domain Database (CDD) (19), and the potential biological functions of the region where the polymorphism sites were located was discussed. In addition, the potential eNOS signaling pathway and the effects of structural changes on the pathway activity were analyzed by using the Kyoto Encyclopedia of Genes and Genomes (KEGG) pathway database (20) (http://www.genome.jp/kegg/pathway.html).

Statistical analysis. All data were analyzed by SPSS 19.0 (IBM Corp., Armonk, NY, USA). Data are presented as the mean \pm standard deviation, and the comparisons between two groups were performed by Student's t-test. The comparison of enumeration data in two groups (including sex, smoking, hypertension and genotype, Tables I, II and III) was performed 
Table I. Comparison of subject characteristics between the CHD and control groups.

\begin{tabular}{|c|c|c|c|}
\hline Characteristic & CHD group (n=234) & Control group $(\mathrm{n}=228)$ & P-value \\
\hline Age, years & $34.2 \pm 3.7$ & $35.7 \pm 2.6$ & 0.73 \\
\hline Sex & & & 0.84 \\
\hline Male & 198 & 195 & \\
\hline Female & 36 & 33 & \\
\hline BMI, $\mathrm{kg} / \mathrm{m}^{2}$ & $25.1 \pm 3.2$ & $24.6 \pm 3.4$ & 0.41 \\
\hline Smoking & 37 & 34 & 0.69 \\
\hline Hypertension & 47 & 51 & 0.52 \\
\hline Diabetes & 24 & 22 & 0.61 \\
\hline Family history of CHD & 39 & 24 & 0.23 \\
\hline \multicolumn{4}{|l|}{ Medication } \\
\hline Nitric acid ester & 60 & NA & NA \\
\hline$\beta$-blocker & 72 & NA & NA \\
\hline Antithrombotic & 63 & NA & NA \\
\hline Statins & 87 & NA & NA \\
\hline Other & 28 & NA & NA \\
\hline
\end{tabular}

For smoking, hypertension and diabetes categories: The individuals who smoked at the time of the study or previously in their lifetime and who had a history of smoking but quit smoking for less than a year were all considered smoking; individuals who had hypertension or diabetes at the time of the study or prior to the study were categorized as hypertension or diabetes. The number of individuals in each group that were smokers or had hypertension or diabetes are presented, respectively. Within the medication category, the 'other' group included calcium channel blockers and renin-angiotensin system inhibitors. Certain patients were treated with two drugs and others were treated with interventional therapy and medication. BMI, body mass index; CHD, coronary heart disease; NA, not applicable.

by Chi-squared $\left(\chi^{2}\right)$ test. $\mathrm{P}<0.05$ was considered to indicate a statistically significant difference. Hardy-Weinberg equilibrium was used to analyze whether samples in each group were representative of the population and $\mathrm{P}>0.05$ was considered to indicate that the sample was representative. The differences in the distribution of genotype and alleles between the two groups were compared by the $\chi^{2}$ test and binary logistic regression was performed to analyze the association between genotype and disease risk, and the odds ratio (OR) value was presented to indicate the relative risk.

\section{Results}

Subject characteristics. The characteristics of the control and CHD groups are presented in Table I. There were no statistically significant differences in age, sex, body mass index, smoking, diabetes, hypertension or family history of CHD between the CHD and control groups $(\mathrm{P}>0.05)$.

Distribution of genotype and allele frequency of the G894T site. As demonstrated in Table II, the distribution of genotype in the control and CHD groups conformed to the Hardy-Weinberg equilibrium $(\mathrm{P}>0.05)$, which indicates that the samples were typically representative. The results for logistic regression following adjustment for other factors, including age, sex, body mass index and smoking, are presented in Table III. Significant differences in the distribution of genotype and allele frequency between the two groups were observed $(\mathrm{P}<0.05)$, and the risk of CHD in the GT genotype group was 1.867 times the risk in the GG genotype group, while the risk of CHD in TT and GT + TT groups was 2.075 and 1.889 times the risk in the GG group, respectively $(\mathrm{P}<0.05$; Table III). Furthermore, the risk of CHD associated with the eNOS 894T allele was 1.749 times the risk associated with the eNOS $894 \mathrm{G}$ allele $(\mathrm{P}<0.05$; Table III).

Partial structural modeling of the G894T locus. The eNOS G894T gene polymorphism leads to a change in the amino acid located at the 289th amino acid position, changing from glutamic acid (Glu/E) to aspartic acid (Asp/D), and the result of modeling is presented in Fig. 2 as obtained from QUARK (14) and the Cn3D 4.3.1 viewer software (http://www.ncbi.nlm. nih.gov). When the 289th amino acid was changed from Glu to Asp, the tertiary structure of the short-peptide changed from a random coil to an $\alpha$-helix, indicating that the local structure of the protein in different genotypes was different, which may result in alterations to the protein function.

Analysis of structural domain. The structural domain of eNOS was analyzed by SMART and results are presented in Fig. 3. The G894T site was located in the low complexity region at the 287-321th amino acid of eNOS (Fig. 3A), which indicated that the region may be involved in flexible binding associated with specific functions and important in determining binding properties and biological roles (21). Furthermore, CDD analysis demonstrated that G894T was located in the active region of the eNOS enzyme, which binds to the substrate L-arginine, zinc, the cofactor heme, tetrahydrobiopterin and the C-terminal electron supplying reductase region. Therefore, it may be hypothesized that the G894T polymorphism may 
Table II. Analysis of the distribution of genotype frequency by the Hardy-Weinberg equilibrium.

A, Comparison of actual and theoretical genotype frequencies in the CHD group

\begin{tabular}{lcccc}
\hline & \multicolumn{3}{c}{ Genotype } \\
\cline { 2 - 5 } Actual/theoretical genotype frequency & GG & GT & TT & Comparison \\
\hline $\begin{array}{l}\text { Actual frequency (n) } \\
\text { Theoretical frequency }\end{array}$ & $\begin{array}{c}67.1(157) \\
66.7\end{array}$ & $\begin{array}{c}29.1(68) \\
30.0\end{array}$ & $\begin{array}{c}3.8(9) \\
\chi^{2}=0.160, \\
\mathrm{P}=0.923\end{array}$ \\
\hline
\end{tabular}

$\mathrm{B}$, Comparison of actual and theoretical genotype frequencies in the control group

\begin{tabular}{lcccc}
\hline & \multicolumn{3}{c}{ Genotype } & \\
\cline { 2 - 4 } Actual/theoretical genotype frequency & GG & GT & TT & Comparison \\
\hline Actual frequency (n) & $79.4(181)$ & $18.4(42)$ & $2.2(5)$ & $\chi^{2}=0.434$, \\
Theoretical frequency & 78.5 & 20.2 & 1.3 & $\mathrm{P}=0.805$ \\
\hline
\end{tabular}

CHD, coronary heart disease.

Table III. The association between the eNOS G894T polymorphism and the risk of CHD in young individuals.

\begin{tabular}{|c|c|c|c|c|}
\hline Genotype/allele frequency & Control group $(\mathrm{n}=228)$ & CHD group $(n=234)$ & OR $(95 \% \mathrm{CI})$ & P-value \\
\hline \multicolumn{5}{|l|}{ Genotype frequency (\%) } \\
\hline GG & $181(79.4)$ & $157(67.1)$ & 1 & NA \\
\hline GT & $42(18.4)$ & $68(29.1)$ & $1.867(0.892-3.05)$ & 0.034 \\
\hline TT & $5(2.2)$ & $9(3.8)$ & $2.075(1.132-4.865)$ & 0.011 \\
\hline $\mathrm{GT}+\mathrm{TT}$ & $47(20.6)$ & $77(32.9 \%)$ & $1.889(0.902-3.14)$ & 0.029 \\
\hline \multicolumn{5}{|l|}{ Allele frequency (\%) } \\
\hline $\mathrm{G}$ & $404(88.6)$ & $382(81.6)$ & 1 & NA \\
\hline $\mathrm{T}$ & $52(11.4)$ & $86(18.4)$ & $1.749(0.847-2.874)$ & 0.013 \\
\hline
\end{tabular}

The control and CHD groups consisted of 228 and 234 individuals, respectively. P-values indicate statistical comparisons between the OR (95\% CI) values for GT, TT and GT + TT genotype frequencies vs. the GG genotype frequency, or between the OR (95\% CI) values for the T allele frequency vs. the G allele frequency. In addition, genotype (GG, GT and TT) and allele (G and T) frequencies between the control and CHD group were analyzed by the $\chi^{2}$ test, with the following results obtained: Genotype frequencies, $\chi^{2}=8.916$ and $\mathrm{P}=0.012$; allele frequencies, $\chi^{2}=8.434$ and $\mathrm{P}=0.004$. CHD, coronary heart disease; OR, odds ratio; CI, confidence intervals; NA, not applicable.

change the partial structure of the protein and be one of the major functional sites in the active region of the eNOS enzyme. As a result of altered structure of the active region of eNOS, the activity of enzyme may subsequently be altered by affecting its ability to bind to substrates, thus affecting biological functions.

Signaling pathway associated with eNOS and CHD. KEGG pathway analysis demonstrated that eNOS is involved in the processes of atherosclerosis and arginine and proline metabolism. In addition, eNOS was demonstrated to be a key enzyme involved in the conversion of L-arginine to $\mathrm{NO}$ in endothelial cells (22); eNOS catalyzes the generation of NO, which activates guanylate cyclase in vascular smooth cells to increase cyclic (c)GMP levels, which subsequently relaxes the vascular
A

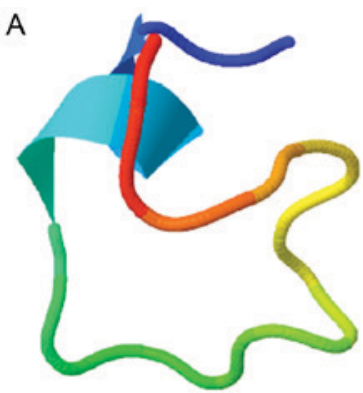

Figure 2. Tertiary structure diagram for the partial structure of the G894T endothelial nitric oxide synthase polymorphism, which obtained using QUARK and the Cn3D viewer software. (A) Protein structure when the 298th amino acid was glutamic acid. The modeling sequence was 'lplllqapddppelfllppe'. (B) Protein structure when the 298th amino acid was aspartic acid. The modeling sequence was 'Iplllqapdeppelfllppe'. 

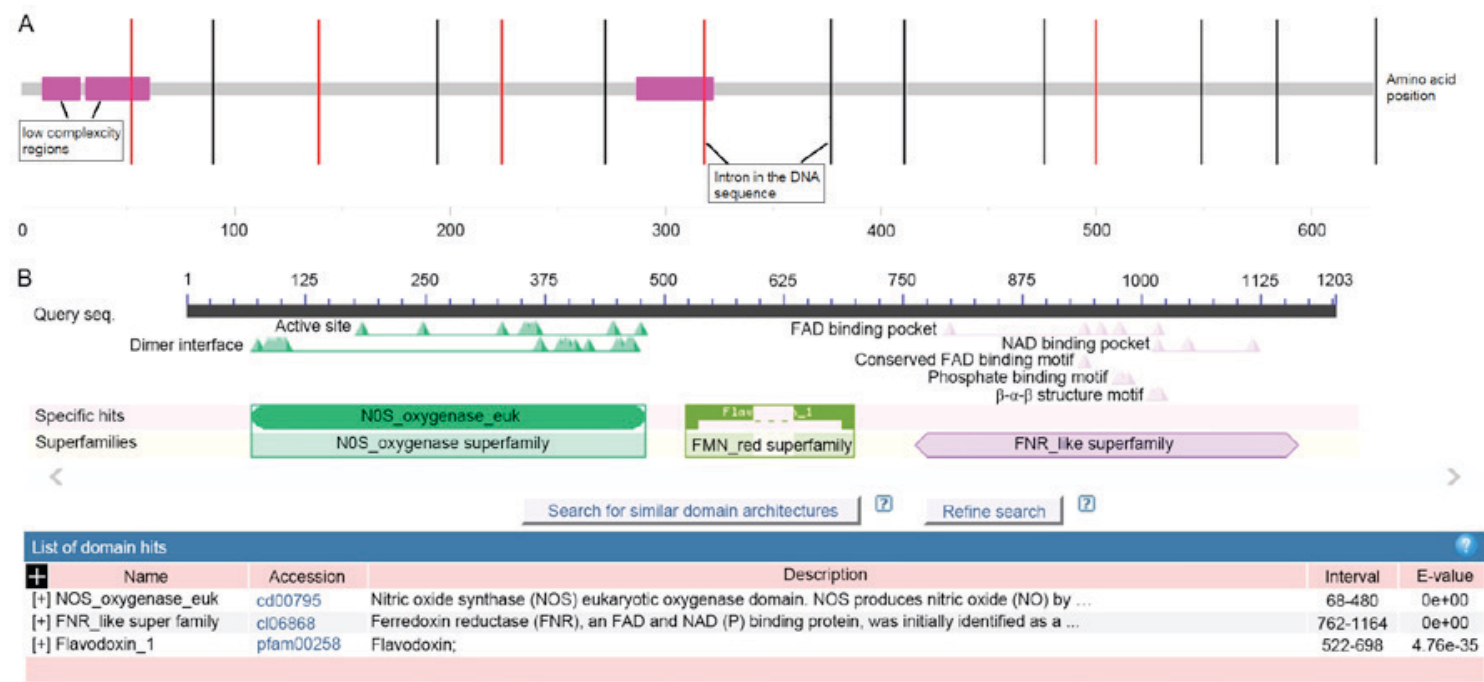

Figure 3. Analysis of structural domains of the eNOS protein. (A) Analysis of the eNOS protein structural domain by SMART. Pink boxes represented low complexity regions; red and black lines indicated the X-axis. (B) Analysis of the eNOS protein structural domain by NCBI Conserved Domain Database. eNOS, endothelial nitric oxide synthase.

smooth muscle and leads to vasodilation to regulate the blood pressure, and consequently prevents cardiovascular diseases such as atherosclerosis (23). Therefore, it may be hypothesized that, if the partial structure of eNOS is altered due to the eNOS G894T polymorphism, the activity of the enzyme may be reduced. Upon reduced enzymatic activity, the binding of L-arginine and eNOS may be reduced, which would subsequently reduce the generation of $\mathrm{NO}$ and lead to improper regulation of vasodilatation and vasoconstriction, with altered blood pressure and blood flow, which may reduce superoxide clearance (24). As a result, coronary vascular atherosclerosis and thrombi may develop, which increase the risk of CHD (Fig. 4). Therefore, alterations in the activity of eNOS, levels of NO, activity of guanylate cyclase and levels of cGMP in individuals with the different genotypes for this polymorphism should be determined in further studies to confirm the above hypothesis, which may aid the development of novel drugs for the treatment of CHD at a molecular level.

\section{Discussion}

CHD may be caused by various factors, including genetic and environmental factors (25). At present, CHD incidence is increasing within the Chinese population and the number of young patients with CHD is increasing annually (26). Studies have demonstrated that during the process of atherosclerosis, eNOS gene polymorphisms may affect the activities of the enzyme and be associated with the incidence of CHD $(27,28)$. However, to the best of our knowledge, the association between eNOS gene polymorphisms and CHD in young people has not been previously clarified. The present study compared the distribution of genotypes and allele frequency of the eNOS G894T gene polymorphism between CHD and control groups, analyzed alterations in the protein structure by bioinformatics and further discussed the association between the eNOS G894T polymorphism and the risk of CHD in young individuals, in addition to the potential mechanisms.

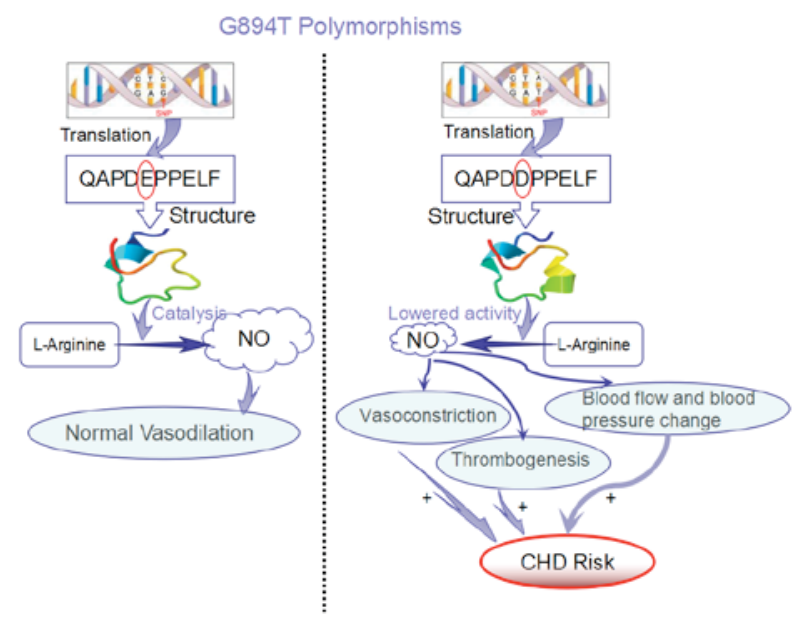

Figure 4. Signaling pathway associated with eNOS and CHD. The potential signaling pathway associated with the development of CHD when the $894 \mathrm{G} \rightarrow \mathrm{T}$ mutation is present, which causes the 289th amino acid glutamic acid $\rightarrow$ aspartic acid missense mutation in the protein sequence. This alteration may theoretically alter the activity of eNOS by affecting its ability to bind to its substrate, L-arginine, and reducing NO levels. Lower NO levels lead to vasoconstriction, subsequently reduced blood flow, and increased blood pressure and risk of thrombogenesis, which all contribute to an increased risk of CHD. E and D highlighted by the red circles indicate glutamic acid and aspartic acid amino acids, respectively, at the 289th amino acid position. eNOS, endothelial nitric oxide synthase; CHD, coronary heart disease; NO, nitric oxide; E, glutamic acid; D, aspartic acid.

The association between the eNOS G894T gene polymorphism and the risk of premature CHD has been reported by various studies globally, however, results obtained in the same region and for similar ethnic groups were different $(15,29,30)$. A study by Colombo et al (31) included 315 Italians with 201 CHD cases and 114 controls, and the results demonstrated that the incidence and severity of CHD was associated with the G894T polymorphism. By contrast, a study on an Italian population by Rossi et al (32) indicated that CHD was independent of the eNOS G894T polymorphism. In China, He et al (33) 
reported that differences in the T allele frequency of the G894T polymorphism between the CHD and control groups were statistically significant $(\mathrm{P}<0.05)$ in a study where participants were from Henan province. However, Liang et al (34) demonstrated that the genotype TT was significantly different in the CHD and control groups (OR, 8.50, $\mathrm{P}<0.05)$, while differences in the $\mathrm{T}$ allele frequency of the G894T polymorphism between the CHD and control groups was not statistically significant. The results of the present study indicated that the differences in the distribution of genotype and allele frequency of the eNOS G894T gene polymorphism between the young CHD and control groups were statistically significant, which indicated that the incidence of CHD in young people may be associated with the eNOS G894T gene polymorphism. The risk of CHD associated with the GT genotype was 1.867 times the risk associated with the GG genotype, while the risk for TT and GT + TT genotypes was 2.075 and 1.889 times the risk for the GG genotype, respectively. In addition, the risk of CHD associated with the eNOS 894T allele was 1.749 times the risk associated with the eNOS $894 \mathrm{G}$ allele $(\mathrm{P}<0.05)$. These results indicated that individuals with the T allele of the G894T polymorphism may be at a higher risk of CHD.

The result of structure modeling based on the amino acid sequence demonstrated that the protein structure of the G894T polymorphism was different prior to and following mutation, which led to partial structures in the active structural domain of eNOS being altered from a random coil to an $\alpha$-helix. Therefore, it was hypothesized that this altered structure may influence the activity of eNOS and the binding of eNOS with L-arginine, consequently decreasing the generation of NO. Low NO levels restrict vasodilation, reduce blood flow, affect the clearing of superoxide in the blood, promote blood platelet adherence and reduces the oxidation of low-density lipoprotein cholesterol. Therefore, reduced NO levels accelerates the formation of coronary atherosclerosis and thrombi, consequently increasing the incidence of CHD (35). Although the observed structural difference would be present in all individuals that possess the 894T allele, and not just young individuals with this allele, 894T may be an important risk factor for early-onset CHD. Therefore, it may be more obvious in young patients with CHD that the eNOS G894T polymorphism is associated with the development of CHD, compared with older patients.

In conclusion, the occurrence and development of CHD in young people may be associated with the eNOS G894T gene polymorphism. The potential mechanism may be that the 894 $\mathrm{G} \rightarrow \mathrm{T}$ mutation, which causes the 289 th amino acid $\mathrm{Glu} \rightarrow \mathrm{Asp}$ missense mutation in the protein sequence, leads to alterations in the functional structure domain of partial structures in eNOS, influencing the activity of eNOS and the binding of substrates to eNOS and reducing the generation of NO, consequently leading to the incidence of CHD. However, there certain limitations are associated with the present study. Firstly, only one polymorphism site was analyzed in the current study, and the interaction between G894T and other polymorphism sites in eNOS has not been investigated. Previous studies have indicated that additional polymorphism sites in eNOS, including rs2070744 T786C and eNOS4a/4b 27 bp variable number tandem repeat, may also be associated with $\mathrm{CHD}(22,36,37)$; therefore, whether the G894T polymorphism may lead to CHD via combined action with other polymorphism sites in eNOS requires further investigation. Additionally, the mechanism was analyzed by a bioinformatics tool and, although structural alterations were identified, the effect of these structural alterations on eNOS activity requires verification, which may illustrate the association between the G894T polymorphism and the risk of CHD more clearly. Furthermore, although the risk of CHD was analyzed in the current study, the association between the G894T and the efficacy of drugs for the treatment of CHD was not determined. Therefore, investigation into the association between the eNOS polymorphism and the efficacy of drugs for the treatment of CHD is required to determine whether the polymorphism affects drug sensitivity or multi-drug resistance may be of clinical importance for the treatment of CHD.

\section{References}

1. Lv H and Guo Y: Progress in the treatment of coronary heart disease. Jin Ri Jian Kang 15: 149, 2016 (In Chinese).

2. Batty GD, Shipley M, Smith GD and Kivimaki M: Long term risk factors for coronary heart disease and stroke: Influence of duration of follow-up over four decades of mortality surveillance. Eur J Prev Cardiol 22: 1139-1145, 2015.

3. Che J, Li G, Shao Y, Niu H and Shi Y: An analysis of the risk factors for premature coronary artery disease in young and middle-age Chinese patients with hypertension. Exp Clin Cardiol 18: 89-92, 2013.

4. Yao HM, Sun TW, Wan YD, Zhang XJ, Fu X, Shen DL, Zhang JY and Li L: Domestic versus imported drug-eluting stents for the treatment of patients with acute coronary syndrome. World J Emerg Med 5: 175-181, 2014.

5. Tolstrup JS, Hvidtfeldt UA, Flachs EM, Spiegelman D, Heitmann BL, Bälter K, Goldbourt U, Hallmans G, Knekt P, Liu S, et al: Smoking and risk of coronary heart disease in younger, middle-aged, and older adults. Am J Public Health 104: 96-102, 2014

6. Ben AM, Messaoudi S, Ezzine H and Mahjoub T: Contribution of eNOS variants to the genetic susceptibility of coronary artery disease in a Tunisian population. Genet Test Mol Biomarkers 19: 203-208, 2015.

7. Han Xu WG: Research progress in pathogenesis of coronary heart disease and its correlation with endothelin and nitric oxide. China Medical Herald: 167-169, 2014.

8. Rochette L, Lorin J, Zeller M, Guilland JC, Lorgis L, Cottin Y and Vergely C: Nitric oxide synthase inhibition and oxidative stress in cardiovascular diseases: possible therapeutic targets? Pharmacol Ther 140: 239-257, 2013.

9. Shu X, Keller TT IV, Begandt D, Butcher JT, Biwer L, Keller AS, Columbus L and Isakson BE: Endothelial nitric oxide synthase in the microcirculation. Cell Mol Life Sci 72: 4561-4575, 2015.

10. Levinsson A, Olin AC, Bjorck L, Rosengren A and Nyberg F: Nitric oxide synthase (NOS) single nucleotide polymorphisms are associated with coronary heart disease and hypertension in the INTERGENE study. Nitric Oxide 39: 1-7, 2014.

11. Chang FJ, Yuan HY, Hu XX, Ou ZJ, Fu L, Lin ZB, Wang ZP, Wang SM, Zhou L, Xu YQ, et al: High density lipoprotein from patients with valvular heart disease uncouples endothelial nitric oxide synthase. J Mol Cell Cardiol 74: 209-219, 2014.

12. Na Z and Jin-Fei Hu: A case-control study on the association between single nucleotide polymorphism of eNOS gene T-786C and G894T and the risk of coronary heart disease. Zhejiang J Prevent Med 28: 9-12, 2016.

13. Azzam N, Zafrir B, Fares F, Smith Y, Salman N, Nevzorov R and Amir O: Endothelial nitric oxide synthase polymorphism and prognosis in systolic heart failure patients. Nitric Oxide 47: 91-96, 2015.

14. Vecoli C: Endothelial nitric oxide synthase gene polymorphisms in cardiovascular disease. Vitam Horm 96: 387-406, 2014.

15. Zhang K, Bai P, Shi S, Zhou B, Wang Y, Song Y, Rao L and Zhang L: The G894T polymorphism on endothelial nitric oxide synthase gene is associated with increased coronary heart disease among Asia population: Evidence from a Meta analysis. Thromb Res 130: 192-197, 2012.

16. Dawber TR, Moore FE and Mann GV: II. coronary heart disease in the framingham study. Int J Epidemiol 44: 1767-1780, 2015. 
17. Xu D and Zhang Y: Ab initio protein structure assembly using continuous structure fragments and optimized knowledge-based force field. Proteins 80: 1715-1735, 2012.

18. Letunic I, Doerks T and Bork P: SMART: Recent updates, new developments and status in 2015. Nucleic Acids Res 43 (Database Issue): D257-D260, 2015.

19. Marchler-Bauer A, Bo Y, Han L, He J, Lanczycki CJ, Lu S, Chitsaz F, Derbyshire MK, Geer RC, Gonzales NR, et al: CDD/SPARCLE: Functional classification of proteins via subfamily domain architectures. Nucleic Acids Res 45: D200-D203, 2017.

20. Kanehisa M, Furumichi M, Tanabe M, Sato Y and Morishima K: KEGG: New perspectives on genomes, pathways, diseases and drugs. Nucleic Acids Res 45: D353-D361, 2017.

21. Coletta A, Pinney JW, Solis DY, Marsh J, Pettifer SR and Attwood TK: Low-complexity regions within protein sequences have position-dependent roles. BMC Syst Biol 4: 43, 2010.

22. Yang Y, Du K, Liu Z and Lu X: Endothelial nitric oxide synthase (eNOS) 4b/a gene polymorphisms and coronary artery disease: Evidence from a meta-analysis. Int J Mol Sci 15: 7987-8003, 2014.

23. Loukanov T, Hoss K, Tonchev P, Klimpel H, Arnold R, Sebening C, Karck M and Gorenflo M: Endothelial nitric oxide synthase gene polymorphism (Glu298Asp) and acute pulmonary hypertension post cardiopulmonary bypass in children with congenital cardiac diseases. Cardiol Young 21: 161-169, 2011.

24. Yousry SM and Sedky Y: Relation of Endothelial Nitric Oxide Synthase (eNOS) Genetic polymorphisms and pulmonary hypertension in egyptian children with congenital heart disease Sherif Mohamed. J Am Sci 10: 124-130, 2014.

25. Wang D, Li W, Cui X, Meng Y, Zhou M, Xiao L, Ma J, Yi G and Chen W: Sleep duration and risk of coronary heart disease: A systematic review and meta-analysis of prospective cohort studies. Int J Cardiol 219: 231-239, 2016.

26. Yang WX, Yang Z, Wu YJ, Qiao SB, Yang YJ and Chen JL: Factors associated with coronary artery disease in young population (age <40): Analysis with 217 cases. Chin Med Sci J 29: 38-42, 2014.

27. Seckin S, Emrah B, Biyik I, Emre A, Burak T, Azmi S, Omer C and Sinan D: 786T/c endothelial nitric oxide synthase gene polymorphism and coronary collateral circulation. Postepy Hig Med Dosw (Online) 70: 80-85, 2016.

28. Xin W: A study on the association between intracranial atherosclerosis and carbonyldiamide and the polymorphism of genes related to nitric oxide synthetic pathway. Southern Medical University 12: 37-40, 2013
29. Xueping J: Study of eNOS Gene G894T polymorphism in CHD patients of multi-ethnic group of xingjiang. Chin J Health Lab Technol: 1460-1461, 2010.

30. Chen C and Jiang X: Study on polymorphism of eNOS gene G894T in Kazak patients with CHD in Xinjiang. J Clin Exp Med 9: 451-499, 2010.

31. Colombo MG, Andreassi MG, Paradossi U, Botto N, Manfredi S, Masetti S, Rossi G, Clerico A and Biagini A: Evidence for association of a common variant of the endothelial nitric oxide synthase gene (Glu298 $\rightarrow$ Asp polymorphism) to the presence, extent, and severity of coronary artery disease. Heart 87: 525-528, 2002.

32. Rossi GP, Cesari M, Zanchetta M, Colonna S, Maiolino G, Pedon L, Cavallin M, Maiolino P and Pessina AC: The T-786C endothelial nitric oxide synthase genotype is a novel risk factor for coronary artery disease in Caucasian patients of the GENICA study. J Am Coll Cardiol 41: 930-937, 2003.

33. He Y, Yang D and Yu H: Research on Correlativity of 27 bpVNTR and G894T polymorphisms of endothelial nitric oxide synthase gene and coronary heart disease in henan han population. China J Modern Med: 523-527, 2008.

34. Liang Q, Dong Y and Yang X: The research of AC angiotensinogen and endothelial nitric oxide synthase gene polymorphisms in predisposition to CHD with gene chip technology. Hebei Med: 403-406, 2006

35. Faure C, Leveille P, Dupont C, Julia C, Chavatte-Palmer P; Alifert Group, Sutton A and Levy R: Are superoxide dismutase 2 and nitric oxide synthase polymorphisms associated with idiopathic infertility? Antioxid Redox Signal 21: 565-569, 2014.

36. Kumar GR, Spurthi KM, Kumar GK, Aiyengar TM, Chiranjeevi P, Nivas S, Anuradha C, Swathi B, Sahu SK, Ali A and Rani HS: Genetic polymorphisms of eNOS (-786T/C, Intron $4 \mathrm{~b} / 4 \mathrm{a} \& 894 \mathrm{G} / \mathrm{T}$ ) and its association with asymptomatic first degree relatives of coronary heart disease patients. Nitric Oxide 60: 40-49, 2016.

37. Liu D, Jiang Z, Dai L, Zhang X, Yan C and Han Y: Association between the-786T $>\mathrm{C}$ 1polymorphism in the promoter region of endothelial nitric oxide synthase (eNOS) and risk of coronary artery disease: A systematic review and meta-analysis. Gene 545: 175-183, 2014.

This work is licensed under a Creative Commons Attribution-NonCommercial-NoDerivatives 4.0 International (CC BY-NC-ND 4.0) License. 